\title{
Managing a non-linear scenario - A narrative evolution
}

\author{
Sandy Louchart ${ }^{1}$, Ruth Aylett ${ }^{2}$ \\ ${ }^{1}$ Centre for Virtual Environments, University of Salford, Salford M5 4WT \\ s.louchart@salford,ac.uk \\ ${ }^{2}$ MACS, Heriot-Watt University, Riccarton, Edinburgh EH10 4AS \\ ruth@macs.hw.ac.uk
}

\begin{abstract}
This paper examines the causes and consequences of the narrative paradox phenomenon widely observed in VR. We present an alternative approach to virtual and interactive storytelling in the form of the emergent narrative concept, together with an implementation of a subset of these ideas in the FearNot! demonstrator.
\end{abstract}

\section{Introduction}

The continuously evolving and developing technology of Virtual Reality (VR) and the rapid technical development of real-time interactive computer graphics has produced a new expressive medium. However, Interactive and Virtual Storytelling (IS/VS) have yet to conclusively address several fundamental and critical issues. What is the role of the user in interactive dramas? What shape and form should they take? How should a story be considered? How should it be articulated in regard to interaction from the user(s) or artificial entities? And how can we manage a non-linear scenario?

In this paper we seek to address these issues by dealing with the narrative paradox [1] generated by the conflict between the interactive freedom that is a basic characteristic of the medium [2] and the structure required by traditional conceptions of plot. Indeed, we question the very validity of the Aristotelian plot [3] as a useful principle in the face of interactivity, suggesting that it has dramatically altered the inherent nature of narrative in VR.

First we present the Emergent Narrative (EN) concept as an alternative approach to conventional thinking, and as a potential solution to the narrative paradox. We will then consider the issues arising from the introduction of interactive elements within a narrative frame and its repercussions on the way interactive narratives might be perceived. Finally, we will investigate the potential of the Emergent Narrative approach to successfully manage and articulate interactive dramas, citing a small-scale experiment in a constructed system. 


\section{Emergent Narrative as a source of story}

Though the merging of emergent and narrative elements as a potential solution to the narrative paradox should be seen as a novel approach, the concepts of emergent structures and bottom-up designs have been studied and developed for many years [4] In particular, these concepts have recently been widely applied to the entertainment industry, in what has been popularly described as "reality TV". Although not advertised as such, television shows such as "Big Brother ${ }^{\mathrm{TM}}$ " in the UK, in which stories emerge directly from the interactions between their different protagonists (i.e. contestants, audience, host), can be recognised as emergent narrative forms.

These programmes, whether we personally enjoy them or not, show that non-linear character-based applications can produce entertaining and meaningful stories. However, one should recognise that these shows are entertaining because they are appropriately edited and that they provide two totally different experiences, depending on whether the user is a participant or a spectator. In this case the spectator perspective is typically more entertaining than that of the participants. In fact, the general feeling inside the confines of the show is often one of boredom, and participants in different series have expressed the desire to leave for that exact reason. The participants are not there to be entertained, they are the entertainers

In this application of the emergent narrative concept to the television medium, the audience is the prime target and represents the population that should be entertained; the participants are offered the promise of a large sum of money and television exposure to encourage them to play their role in the making of the show. The narrative is managed as a process where participants create the story through their interactions with each other, The role exercised in this case by the programme production team could be related to that carried out by a human Game-Master (GM) in non computerbased Role Playing Games (RPG). We earlier identified [5] that one of the most important tasks carried out by the GM was to create, with a rich level of detail, the different worlds, props and characters necessary for the game or campaign and their different interactions and relations with each other that would generate potentially interesting situations. The main aim is for these particular interactions or predetermined events, is to provoke dramatic reactions within the players. This role is indeed perfectly assumed by the programme's production team.

In any character-based drama or application, the choice and definition of the main protagonists, the contestants in this case, is essential to the generation of interesting and potentially dramatic events and ultimately stories or micro-stories. The contestants are selected with great care in relation to their personalities, beliefs, social backgrounds, mental strength or sexual orientation. By selecting contestants with conflicting characteristics, the production team increases the chances of interactions between them creating dramatic events and potentially interesting narrative elements. In most cases such interactions arise from emotions such as tension, frustration, passion or temper, resulting in diverse emergent conflicts and alliances. 
The world environment itself is also carefully designed to foster these emotions and deny the contestants any possibility of escaping the constant pressure surrounding them, for example through rules preventing contestants from entertaining themselves by any means other than interacting with each other (i.e. writing, reading). Although, the situations generated in the show emerge from direct interactions between contestants and could not possibly have been planned in detail beforehand, certain likely or recurrent scenarios are taken into account. For instance, it makes perfect sense to design a remote area where contestants can experience relative privacy, as long as there are enough cameras and microphones in that area to cover every possible angle. This allows for interactions that are generally seen as private or secretive such as conspiracy, passion or private arguments.

While design is prior to the performance itself, there are also elements of performance-time control. These are limited to the introduction of pre-determined events that establish or create situations likely to generate interesting actions or reactions from the participants. For instance a daily task in which one half of the participants is given food and the other starved for a day creates a certain tension in the group resulting in a higher chance of dramatic interactions. Thus the production team, acting as GM, manages the intensity and the general mood or feeling of the performance from a character perspective to produce emotionally engaging situations for the audience. However, this format does not suffice in itself to provide an entertaining programme that would trigger audience interest sufficiently for them to watch the programme on a regular basis. A cleverly edited footage is thus presented regularly summarising the activity of the contestants and reporting in a dramatic fashion on their feelings, moods, outbursts or attitudes towards each other.

Although such programmes are good applications of the emergent narrative approach, they embody only one form of the concept applied to one particular medium (i.e. television). Moreover since the target is not actively participating in the show, it does not bring any direct solution to the narrative paradox. However, there is much to learn from this particular type of EN and its study can help in shaping a suitable form adapted to immersive interactive media. Several major alterations must be implemented if one is to adapt the EN theory to VR in order to move from a spectating form to the performative one appropriate to an immersed user.[Table 1]. 


\begin{tabular}{|c|c|c|}
\hline \multicolumn{3}{|c|}{ Emergent Narrative forms } \\
\hline & Spectating form & Performative form \\
\hline Medium & Television & Virtual Reality \\
\hline Target audience & General public & User(s) \\
\hline User role & Inactive (mainly) & Active - participating \\
\hline Emergent source & Contestants & User(s) and virtual agents \\
\hline Time constraint & 1 hour summary & Real-time \\
\hline Cast & Rich conflicting characters & Rich conflicting characters \\
\hline Managing constraints & $\begin{array}{l}\text { Public interest } \\
\text { Intensity of the show } \\
\text { Contestants, moods, emotions and } \\
\text { feelings }\end{array}$ & $\begin{array}{l}\text { User interest } \\
\text { Feeling of immersion } \\
\text { Intensity of the performance } \\
\text { User mood, emotions and feeling }\end{array}$ \\
\hline Drama manager & Authoritative and visible & Distributed and hidden (in agents) \\
\hline Dramatic tools & $\begin{array}{l}\text { Narrative and situational events, } \\
\text { editing }\end{array}$ & Narrative and situational events \\
\hline
\end{tabular}

Table 1: Performative and Spectating Emergent Narrative forms

The role and nature of the user is crucial for two somewhat different forms of emergent narrative. If the user is part of an audience and does not have any active role within the performance, the focus of attention will be on providing user entertainment, even if this is to the detriment of the participant's experience. The "spectating" form of EN aims at providing something interesting to watch, look at or read. This is not dissimilar to organic improvisational drama, where the director, in rehearsals, creates certain situations for actors to improvise. The best and most interesting interactions will be then used as raw creative material for a theatrical piece.

The "performative" form of the EN approach is different in nature. Since the main centre of attention is a participating and active user, the focus for the performance is to give the user an enjoyable and compelling experience. Thus, the aim is to produce something that is interesting to live and experience rather than watch. Indeed, the whole experience need not produce anything visually presentable or particularly interesting from an external perspective as long it was when lived and performed from an internal perspective.

\section{Re-evaluating narrative concepts for VR}

We have previously highlighted the need to approach the role of the user according to the nature of the performance or the medium [6]. The shift of attention from spectator to "spect-actor" (i.e. Augusto Boal's terminology - [7]) or participant in an interactive scenario focuses on interactivity and immersion. However, such shift in emphasis cannot be made without a major rethink on the exact nature of stories, their requirements and their definitions. The narrative medium has evolved with the introduction of interactive features or elements - particularly true for VR - while the set of narrative tools at our disposition to analyse, understand and generate such interactive narratives has not been developed with respect to this particular 
characteristic. Although several landmark works [8,9] have been published over the years, work in the domains of IS and VS is still at an experimental stage. Arguably, interactivity represents the most significant technical change in narrative terms since the invention of cinema and requires a new examination of the basic concepts.

STORY The first such concept is that of story itself. How can a story be depicted and executed so as to include interactivity with a user in a virtual environment? A storyline or plot exercises constrains the user's freedom of movement and action within the virtual world, while allowing the user interactive freedom affects the unfolding of the story, hence the narrative paradox.

It appears that once interactivity is involved, story becomes plural. All of the different approaches studied in recent years, branching [10,11,12] or emergent, actually deal with multiple stories. In the case of branching systems, the stories potentially displayed and executed are all instances or variations of a given story, while in emergent concepts, they result from the association of many micro-stories at character level. Although multiple storylines are common in literature, cinema or even theatre, VR presents the major characteristic that changes or alterations made in these sub-stories are orchestrated consciously or not by the user. This makes the execution of an Aristotelian plot a difficult challenge, in terms of timing and outcome from a branching point of view and in terms of formulation, articulation and representation from an emergent perspective.

Although the general format of beginning middle and end can be respected in principle, an emergent approach to storytelling need not apply it in the sense that it focuses more on the actions and paths of individual characters than on an overall general story. Our definition of what makes a story has to be extended and broadened in the face of interactivity to the consideration of narrative as a dynamic process rather than a static structure. Conventional storytelling (i.e. Aristotelian plot) can then be seen as a support tool that can help in bringing depth, meaning and context to a performance, rather than the directed or targeted objective. To a certain extent, though based on actions rather than character development, this technique is very much in use in the computer games industry, particularly when designing adventure games. The story can then be used for generating interesting and contextually correct events while still not interfering with the user's freedom of movement within the story world.

NARRATIVE AUTHORING. Authoring represents a major challenge to this shift in perspective. Authoring, in storytelling terms, is stereotypically the representation of the author's mind, the vision of one person. The author's ability to create interesting stories, characters and narrative events, is coupled with control over the timing, order, rhythm and nature of the different story events and their display. The story, as witnessed by the spectator, is the procession of an appropriately orchestrated narrative vision for dramatic purposes. The idea of an intervening user is incompatible with such an approach since one cannot expect the user to make the right decision for the 
story, at the right moment or even at the right place, unless the user plays a role similar to that of a conventional actor following a script.

However, if one wants the user to be able to exercise a certain level of freedom of movement within the virtual environment, and, therefore avoid the narrative paradox, one has to consider the user as an autonomous actor rather than an actor in the conventional sense of the term. Autonomous actors would play character parts or roles in a scenario while still enjoying the freedom to make their own decisions at times they feel appropriate. This view of the user would allow both behaviours and narrative events to emerge from interactions and to some extent reconcile narrative input and user freedom.

However this forces a change in the whole concept of narrative authoring; the focus of attention should be on the characters and their ability to interact with each other rather than the overall story. Indeed, the role of the author is confined to writing interesting characters with a strong potential for dramatic interaction together with flexible narrative events whose only aim is to set up scenes and situations. If authors are to write for interactive applications in this way, they must let go some of their authorial powers, a change in the opposite direction to film with its increase in control (i.e. camera angles, music soundtrack etc.). The overall story should be regarded as a hypothetical meta-structure not directly experienced by any character, whose aim is to set up interesting or potentially interesting situations.

It becomes essential for the author to be extremely attentive to the inner state of the user, or in more generalised terms, to the characters' internal states. The primacy of dynamic internal states, and specifically that of the user makes the whole process of evaluating a story difficult and even relatively pointless and puts the focus on the character and its emotional activity. The success of a performance results not from the quality of the story in conventional terms, but the level of enjoyment, active and willing participation from the user.

STORIFICATION In conventional narrative forms the engagement of the user is reported indirectly by applause or even global sales; in a participative form it is basic to narrative development. Although one can and should analyse signs of enjoyment or immersion of users via their behaviours, level of activity or response within a performance, essential information for the evaluation of such a narrative approach still remains undisclosed and only known to the users. Some can be retrieved through the use of post-performance questionnaires but the subjective story-as-experienced may remain permanently hidden. A feature of live role-play is the debrief at the end, in which the multiple story experiences of the participants are shared and integrated through the appreciation of larger-scale causal chains than those an individual has directly experienced.

Storyfication [13] is a term that defines the continuous activity of a narrative participant in building a mental picture and developing and testing expectations about the story's outcome and the character's present and future motivations, roles and emotions as the story unfolds in real-time. What separates this process from the 
variant present in spectating is the situated position of the participant - more limited in terms of global understanding, but richer in terms of ability to act.

In the current absence of non-invasive and reliable mechanisms for estimating user emotional state, one can fall back on monitoring external signs of non commitment, as seen in RPGs [5] where the GM constantly tracks the user's activity or behaviour (i.e. suicidal behaviour, lack of activity, clear lack of interest, lack of attention) in order to assess his/her internal state with respect to the performance. Theatre, cinema and literature have shown that the user's internal emotional state can be manipulated to a certain extent via purposely misguiding hints or indications creating the right frame of mind for a particular effect (i.e. suspense, twist, or surprise), and these techniques need to be reanalysed in the context of EN both for the benefit of authoring and narrative management in real time.

\section{Managing an interactive drama - an EN drama-manager}

Story or drama management, is typically where the crunch takes place in interactive narrative. The role of a manager in application of conventional narrative theory is to keep the overall story 'on track' in the face of user actions. Branching narratives avoid the problem by authoring management into the branches. Other approaches involve variants of universal plans [14], whether through the beats of Façade [8] or interacting plan trees [9]. Here management is a navigation issue, but short of anticipating every possible user action in context as in Façade, in which the author faces a combinatorial explosion of possibilities, only certain paths constitute 'the story' and the management dilemma is to how push the user along these paths.

The implication of the arguments advanced so far is that in EN the drama manager should not focus attention on the quality and meaning of the overall story but on the quality of the performance experienced by the different characters (i.e. user, other agents), so that 'staying on track' is no longer an objective. This requires the development of metrics of performance quality, but since it should be measured from the point of view of the different characters, the idea of a distributed story manager within different agents in the world environment is a very natural one.

By equipping characters with an extended action-selection process, in which choice of action is influenced by performance considerations as well as the more usual one of goals and affective state, management would execute below the surface of the visible story and would not disturb the feeling of immersion we are aiming to protect. Global management would then be confined to events exogenous to the characters: entrances, exits, the outcome of unpredictable physical actions (in the absence of comprehensive - and computationally expensive - virtual physics) and, in RPG terms, 'wandering monsters'. Since most of the performance design is directly imputable to the harmonious definition of both the world environment and the characters, as in its RPG counterpart, the role of the drama manager in the EN approach is one of policing the 
boundaries of character roles and introducing situations and narrative events when required [Figure 1].

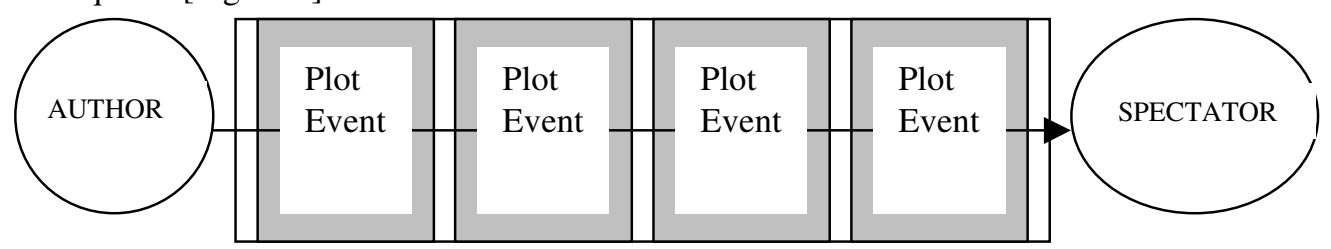

Conventional narrative structure

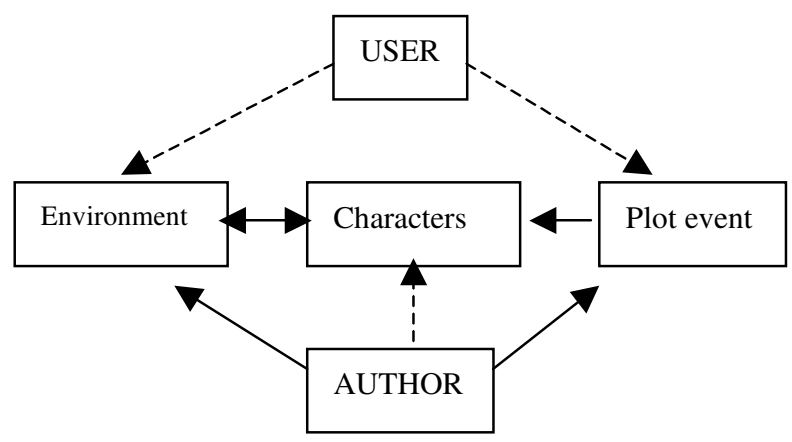

\section{Emergent narrative cycle}

FIGURE1: The emergent narrative articulation

This approach has in fact already been the subject of many applications in the domain of Live RPGs where it has proved successful in adapting scenarios from literary classics such as Shakespeare's Hamlet [15]. In the system we are currently developing, intelligent agents are represented in the story world by a set of goals and potential actions that reflect their personalities. The drama manager is constituted by a set of rules directly extracted from RPG practices. These rules, implemented within the agents' personalities and goal structures, but also within the world framework, are triggered when the performance requires them. They have been designed to respond to the intensity of the performance and monitor the activity and emotional state of each character in the performance.

\section{FearNot! an example of emergent narrative}

The project VICTEC (2002-05), involving five partners in the UK, Germany and Portugal, applied virtual dramas acted out by 3D graphically-embodied characters to 
anti-bullying education $[16,17]$. The aim of the FearNot! (Fun with Empathic Agents Reaching Novel outcomes in Teaching) demonstrator was to allow children to explore what happens in bullying in an unthreatening environment in which they took responsibility for what happened to a victim, without themselves feeling victimized. The child was asked to act as an 'invisible friend', and to give coping advice between episodes which would influence the behaviour of the victim in the next one without undermining its autonomy of action and the child's ability to believe in it as a character with an independent inner life. This interactional structure was inspired by the Forum Theatre approach of by Boal [7] and the child acted as a spect-actor.

Given there are around 7 different pieces of coping advice a child could give, and the order in which they are given before the second or third episode would also have to be taken into account, a branching narrative of the type used successfully in MRE [11] or Carmen's Bright IDEAS [10] seems infeasible. Thus EN, in which action is driven by the characters themselves, is a natural solution to making the victim responsive to the advice the child gives. At the same time, the repetitive nature of bullying, and the fact that it is naturally episodic, does not require too much from the emergent mechanism in terms of dramatic complexity or length. The use of the Forum Theatre approach also means that the emergent mechanism does not have to take user actions directly into account. Thus this was a small-scale application of the EN concept, in which the emotional reactions of the characters fed into their individual actionselection mechanisms. Given also the indeterminacy of physical actions in the system - a character who was pushed might or might not fall over - the EN concept produced a variety of storylines in episodes with outcomes essentially rather unpredictable.

A Stage Manager was required because a choice had to be made about where each new episode was located and which characters were involved in it, as well as any other initial conditions. In addition, there has to be some method of determining when an episode has finished once there is no script encoding this information. Deciding the termination condition is a small-scale example of the performance-monitoring activity discussed more generically above.

\section{Conclusion}

In this paper, we presented the formulation of an emergent narrative approach to interactive storytelling as a potential solution to the recurrent problem of the narrative paradox. We have highlighted the needs for a better consideration of stories, characters, performances and narrative displays in regard to interactivity. We have also introduced the FearNot! demonstrator, we are proposing to use for future scale up and drama-oriented experiments on the emergent narrative concept. Finally, we have presented our strong position on the role we believe the user should play in regard to IS/VS. Our view on the matter reflects our belief on the form in which interactivity 
should also be considered in such systems and our approach of the interactivity issue from an internal-ontological approach as described in Ryan [18].

\section{References}

[1] Aylett, R.S. (1999) "Narrative in Virtual Environments: Towards Emergent Narrative". Papers from the 1999AAAI Fall Symposium, Technical report FS-99-01, AAAAI Press, Menlo Park, 1999 pp83-86.

[2] Aylett, R.S \& Louchart,S (2003) Towards a Narrative Theory for Virtual Reality. (2003) Virtual Reality vol 7 no 1 Dec 2003

[3] Aristotle. 330 B.C. The Poetics of Aristotle, translation and commentary by Stephen Halliwell. Duckworth, 1987.

[4] Cliff, D \& Grand. S. (1999) "The creatures global digital ecosystem," Artificial Life, vol. 5, pp. 77--94, 1999.

[5] Louchart, S, Aylett, R.S (2003) Solving the narrative paradox in VEs - lessons from RPGs In: Intelligent Virtual Agents, $4^{\text {th }}$ International Workshop IVA2003 eds T. Rist, R.Aylett, D.Ballin, LNAI 2792 Springer 2003 ISBN: 3-540-20003-7 pp244-248

[6] Aylett, R.S \& Louchart,S (2003) Towards a Narrative Theory for Virtual Reality. (2003) Virtual Reality vol 7 no 1 Dec 2003

[7] Boal, Augusto. (1979) The Theatre of the Oppressed. New York: Urizen Books, 1979

[8] Michael Mateas, Andrew Stern, Structuring Content within the Facade Interactive Drama Architecture First International Conference on Artificial intelligence and Interactive Digital Media, 2005

[9] Cavazza.M, Charles.F, Lozano.M, Mead.S, Bisquerra.A: Planning formalisms and authoring in interactive storytelling, TIDSE. 2003

[10] Marsella, S.C., Johnson, W.L., \& LaBore, C. (2000). Interactive pedagogical drama. In Proceedings of the 4th International Conference on Autonomous Agents, 2000.

[11] Swartout, W and al. Toward the Holodeck: Integrating Graphics, Sound, Character and Story. Proceedings of 5th International Conference on Autonomous Agents (Montreal, Canada, June 2001).

[12] Riedl M, Young M, From Linear Story Generation to Branching Story Graphs, First International Conference on Artificial intelligence and Interactive Digital Media, 2005

[13] Aylett, R.S. (2000) Emergent Narrative, Social Immersion and "Storification" Proceedings, Narrative Interaction for Learning Environments, Edinburgh, 2000

[14] Schoppers M. Advanced Decision Systems - Universal plans of reactive robots in unpredictable environments. 1987; Proceedings of IJCAI 1987

[15] Grutbildning - http://www.grutbildning.to/

[16] Louchart, S; Aylett, R.S; Paiva, A and Dias J. (2005) Unscripted Narrative for affectively driven characters, First International Conference on Artificial intelligence and Interactive Digital Media 2005

[17] Paiva, A; Dias, J; Aylett, R.S; (2005) Learning by Feeling: Evoking Empathy with Synthetic Characters. Applied Artificial Intelligence, 2005. 19(3-4): p. 235-266

[18] Ryan ML; (2001) Beyond Myth and Metaphor: The case of narrative in digital mediaGame studies - the international journal of game research - Volume. 2001 